\title{
Noncommutativity as a Colimit
}

\author{
Benno van den Berg • Chris Heunen
}

Received: 18 March 2010 / Accepted: 30 January 2011 / Published online: 22 February 2011

(C) The Author(s) 2011. This article is published with open access at Springerlink.com

\begin{abstract}
We give substance to the motto "every partial algebra is the colimit of its total subalgebras" by proving it for partial Boolean algebras (including orthomodular lattices), the new notion of partial $\mathrm{C}^{*}$-algebras (including noncommutative $\mathrm{C}^{*}$-algebras), and variations such as partial complete Boolean algebras and partial $\mathrm{AW}^{*}$-algebras. Both pairs of results are related by taking projections. As corollaries we find extensions of Stone duality and Gelfand duality. Finally, we investigate the extent to which the Bohrification construction (Heunen et al. 2010), that works on partial $\mathrm{C}^{*}$-algebras, is functorial.
\end{abstract}

Keywords Partial Boolean algebra $\cdot$ Partial C*-algebra $\cdot$ Colimit

Mathematics Subject Classifications (2010) $16 \mathrm{~B} 50$ • 18A30 • 46L05 • 46L85 • $06 \mathrm{E} 15 \cdot 81 \mathrm{P} 16$

\section{Introduction}

This paper is intended as a contribution to the Bohrification programme [9], which tries to give a mathematically precise expression to Bohr's doctrine of classical concepts, saying that a quantum mechanical system is to be understood through its classical fragments. On Bohr's view, as understood within this programme, quantum mechanical systems do not allow a 'global' interpretation as a classical system, but they do so 'locally'. The programme essentially makes two claims about these

C. Heunen was supported by the Netherlands Organisation for Scientific Research (NWO). Part of this work was performed while the author visited the IQI at Caltech.

B. van den Berg

Fachbereich Mathematik, Technische Universität Darmstadt, Darmstadt, Germany

C. Heunen $(\bowtie)$

Oxford University Computing Laboratory, Oxford, UK

e-mail: chris.heunen@comlab.ox.ac.uk 
classical 'snapshots'. Firstly, it is only through these snapshots that the behaviour of a system and physical reality can be understood. Secondly, these snapshots contain all the information about the system that is physically relevant.

The main result of [9] (inspired by earlier work of Butterfield, Isham and Döring [5]) is that the collection of these classical snapshots can be seen as forming a single classical system in a suitable topos-its so-called Bohrification; we will briefly recall the details of this construction in Section 7. The implication of this result is that a quantum mechanical system can be seen as a classical one, if one agrees that nothing physically relevant is lost by considering classical snapshots and if one is willing to change the logic from a classical into an intuitionistic one. The question is left open how strong these premises are: how much, if anything, of the information about the original quantum mechanical system is lost by conceding in this way to consider it as a classical system? This article investigates how much information about a quantum mechanical system can be reconstructed from its Bohrification by means of colimits. The fact that quantum mechanical systems can be modelled as noncommutative algebras, and classical systems as commutative ones, explains the title "noncommutativity as a colimit".

Our conceptual contributions to the Bohrification programme are twofold. First, we contend that the programme is most naturally seen as concerned with partial algebras, i.e. sets on which algebraic structure is only partially defined. Such partial algebras are equipped with a binary ('commeasurability') relation, which holds between two elements whenever they can be elements of a single classical snapshot. In this setting, commeasurability is often also called commutativity, since in typical examples commeasurability means commutativity in a totally defined algebraic structure. We will show that a meaningful theory of such partial algebras can be developed. In particular, and this is our second contribution, we will indicate why Bohrification makes sense for partial algebras and use this to investigate the functorial aspects of this construction.

The idea to consider partial algebras is not new. In fact, in their classic paper [14], Kochen and Specker use the language of partial algebras to state their famous result, saying that many algebras occurring in quantum mechanics cannot be embedded (as partial algebras) into commutative ones (see also [17]). Their interpretation of this fact as excluding a hidden variable interpretation of quantum mechanics has remained somewhat controversial. In the Bohrification programme, this result is taken just as a mathematical confirmation of the view that quantum mechanical systems do not allow for global interpretations as classical systems.

The result by Kochen and Specker, together with the fact that Bohrification works for partial algebras, indicates that the Bohrification programme is most naturally developed in the context of partial algebras. This led us to develop a new notion of 'partial $\mathrm{C}^{*}$-algebra', the study of which most of this paper is devoted to. Our main technical result is that such a partial $\mathrm{C}^{*}$-algebra is the colimit of its total (commutative) subalgebras, which explains the relation between a partial algebra and its classical snapshots in categorical terms.

In more detail, the contents of this paper are as follows. First we consider Kochen and Specker's notion of a partial Boolean algebra, as a kind of toy example, and prove our main result for them in Section 2, as well as for the variation of partial complete Boolean algebras. This also makes precise the widespread intuition that an orthomodular lattice is an amalgamation of its Boolean blocks. In this simple setting the theorem already has interesting corollaries, as it allows us to derive an adjunction 
extending Stone duality in Section 3. But our main interest lies with partial $\mathrm{C}^{*}$ algebras,

which Section 4 studies; we also prove the main result for variations, such as $\mathrm{AW}^{*}$-algebras and Rickart $\mathrm{C}^{*}$-algebras in that section. This enables an adjunction extending Gelfand duality and puts the Kochen-Specker theorem in another light in Section 5. The two parallel settings of Boolean algebras and $\mathrm{C}^{*}$-algebras are related by taking projections, as Section 6 discusses. Finally, Section 7 shows that the Bohrification construction works for partial $\mathrm{C}^{*}$-algebras as well: we investigate its functorial properties, and conclude that its essence is in fact (a two-dimensional version of) the colimit theorem. ${ }^{1}$

\section{Partial Boolean Algebras}

We start with recalling the definition of a partial Boolean algebra, as introduced by Kochen and Specker [14].

Definition 1 A partial Boolean algebra consists of a set $A$ with

- $\quad$ a reflexive and symmetric binary (commeasurability) relation $\odot \subseteq A \times A$;

- elements $0,1 \in A$;

- a (total) unary operation $\neg: A \rightarrow A$;

- (partial) binary operations $\wedge, \vee: \odot \rightarrow A$;

such that every set $S \subseteq A$ of pairwise commeasurable elements is contained in a set $T \subseteq A$, whose elements are also pairwise commeasurable, and on which the above operations determine a Boolean algebra structure. ${ }^{2}$

A morphism of partial Boolean algebras is a function that preserves commeasurability and all the algebraic structure, whenever defined. We write PBoolean for the resulting category.

Clearly, a partial Boolean algebra whose commeasurability relation is total is nothing but a Boolean algebra. For another example, if we declare two elements $a, b$ of an orthomodular lattice to be commeasurable when $a=(a \wedge b) \vee\left(a \wedge b^{\perp}\right)$, as is standard, any orthomodular lattice is seen to be a partial Boolean algebra. In this case the above observation about totality becomes a known fact: an orthomodular lattice is a Boolean algebra if and only if any pair of elements is commeasurable [13].

We introduce some notation and terminology. If $A$ is a partial Boolean algebra, then a subset $T$ of pairwise commeasurable elements which is closed under all the algebraic operations of $A$ will be called a commeasurable or total subalgebra. Clearly, a commeasurable subalgebra has the structure of a Boolean algebra. Note that if $A$ is a partial Boolean algebra and $S$ is subset of pairwise commeasurable elements, then there must be a smallest commeasurable subalgebra $T$ that contains $S$ : it has to

\footnotetext{
${ }^{1}$ Both the categories of Boolean algebras and of commutative $\mathrm{C}^{*}$-algebras are algebraic, i.e. monadic over the category of sets [10]. We expect that the definitions and results of the present article can be extended to a more general theory of partial algebra, but refrain from doing so because the two categories mentioned are our main motivation.

${ }^{2}$ Note that this means that $T$ must contain 0 and 1 and has to be closed under $\neg, \wedge$ and $\vee$.
} 
consist of the values of Boolean expressions built from elements of $S$. We denote it by $A\langle S\rangle$.

Given a partial Boolean algebra $A$, the collection of its commeasurable subalgebras $\mathcal{C}(A)$ is partially ordered by inclusion. In fact, $\mathcal{C}$ is a functor PBoolean $\rightarrow$ POrder to the category of posets and monotone functions. Regarding posets as categories, $\mathcal{C}(A)$ gives a diagram in the category PBoolean (in fact, it also defines a diagram in the category Boolean of Boolean algebras). The following proposition lists some easy properties of this diagram.

Proposition 1 Let $A$ be a partial Boolean algebra.

(a) The least element of the poset $\mathcal{C}(A)$ is $A\langle 0\rangle=A\langle 1\rangle=\{0,1\}$.

(b) The atoms of $\mathcal{C}(A)$ are $A\langle a\rangle=\{0, a, \neg a, 1\}$ for nontrivial $a \in A$ (an element $p$ of a poset with least element 0 is an atom when there are no elements $x$ such that $0<x<p$ ).

(c) Two total subalgebras $S$ and $T$ have a common upper bound in $\mathcal{C}(A)$ if and only if all elements of $S$ are commeasurable with all the elements of $T$.

(d) $A$ is a (total) Boolean algebra if and only if the poset $\mathcal{C}(A)$ is filtered (meaning that any two elements have an upper bound). In that case, $A$ is the largest element of the poset $\mathcal{C}(A)$.

Proof Parts (a) and (b) are easy to show and therefore we omit their proofs.

To see (c), observe that if total subalgebras $S$ and $T$ have a common upper bound $U$, then all elements of $S$ are commeasurable with all elements in $T$, because all elements of $S$ and $T$ belong to the commeasurable subalgebra $U$. Conversely, if all elements of $S$ are commeasurable with those of $T$, then $A\langle S \cup T\rangle$ is an upper bound (in fact, the least upper bound) in $\mathcal{C}(A)$ of $S$ and $T$.

If $A$ is total, then $A$ is the top element of $\mathcal{C}(A)$ and hence $\mathcal{C}(A)$ is filtered. If, on the other hand, $\mathcal{C}(A)$ is filtered, then for any two elements $a, b \in A$ the total subalgebras $A\langle a\rangle$ and $A\langle b\rangle$ must have an upper bound, which implies (by (c)) that $a$ and $b$ are commeasurable. This shows (d).

Remark 1 One can show that $\mathcal{C}(A)$ is a directed complete partial order (dcpo), which is algebraic, and is such that for every compact element $x$ the downset $\downarrow x$ is dually isomorphic to a finite partition lattice. The main result of the paper [6] suggests that every such dcpo is the $\mathcal{C}(A)$ of a unique partial Boolean algebra $A$. Whether similar results hold for partial $\mathrm{C}^{*}$-algebras remains to be seen.

We are now ready to prove the first version of our main result.

Theorem 1 Every partial Boolean algebra is a colimit of its (finitely generated) total subalgebras.

Proof Let $A$ be a partial Boolean algebra, and consider its diagram of (finitely generated) commeasurable subalgebras $C$. Define functions $i_{C}: C \rightarrow A$ by the inclusions; these are morphisms of PBoolean by construction. Moreover, they form a cocone; we will prove that this cocone is universal. If $f_{C}: C \rightarrow B$ is another cocone, define a function $m: A \rightarrow B$ by $m(a)=f_{A\langle a\rangle}(a)$. It now follows from the assumption that the $f_{C}$ are morphisms of PBoolean that $m$ is a well-defined morphism, too. To see this, 
we need to show that $m$ preserves commeasurability and the algebraic operations of $A$. We check that $m$ preserves commeasurability, omitting a very similar verification that it also preserves the algebraic operations. So, if $a \odot b$, then also $m(a) \odot m(b)$, since $A\langle a, b\rangle$ is a total subalgebra and

$$
\begin{aligned}
& m(a)=f_{A\langle a\rangle}(a)=f_{A\langle a, b\rangle}(a), \\
& m(b)=f_{A\langle b\rangle}(b)=f_{A\langle a, b\rangle}(b),
\end{aligned}
$$

because the $f_{C}$ form a cocone. One easily verifies that $f_{C}=m \circ i_{C}$, and that $m$ is the unique such morphism.

Kalmbach's "Bundle Lemma" [13] gives sufficient conditions for a family of Boolean algebras to combine into a partial Boolean algebra, so that it could be regarded as a converse of the previous theorem.

Notice that the morphisms of PBoolean are the weakest ones for which the previous theorem holds. For example, even when $A$ and $B$ are orthomodular lattices, the mediating morphism $m: A \rightarrow B$ in the proof of the previous theorem need not be a homomorphism of orthomodular lattices. For a counterexample, consider the function
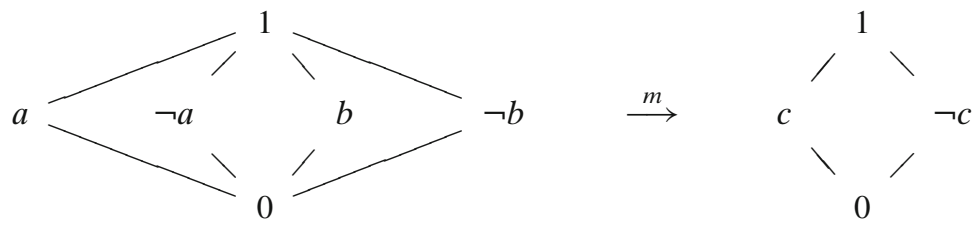

given by $m(0)=0, m(a)=m(b)=c, m(\neg a)=m(\neg b)=\neg c, m(1)=1$. It preserves $0,1, \neg$ and $\leq$. The only commeasurable subalgebras of the domain $A$ are $A\langle 0\rangle, A\langle a\rangle$ and $A\langle b\rangle$, and $m$ preserves $\wedge$ when restricted to those. However, $m(a \wedge b)=m(0)=$ $0 \neq c=c \wedge c=m(a) \wedge m(b)$. (Of course, $\{0, a, b, 1\}$ is a Boolean algebra, but it is not a commeasurable subalgebra, as it does not have the same negation $\neg$ as $A$; see also footnote 2.)

It follows from the previous theorem that the partial Boolean algebra with a prescribed poset of total subalgebras is unique up to isomorphism (of partial Boolean algebras). To actually reconstruct a partial Boolean algebra from its total subalgebras, this should be complemented by a description of colimits in the category PBoolean, as we now discuss.

The coproduct of a family $A_{i}$ of partial Boolean algebras is got by taking their disjoint union, identifying all the elements $0_{i}$, and identifying all the elements $1_{i}$. Notice that elements from different summands $A_{i}$ are never commeasurable in the coproduct. In particular, the initial object $\mathbf{0}$ is the partial Boolean algebra $\{0,1\}$ with two distinct elements.

Incidentally, PBoolean is complete. Products and equalizers of partial Boolean algebras are constructed as in the category of sets; products have commeasurability and algebraic structure defined componentwise, and equalizers have subalgebra structure. Hence the limit of a diagram of Boolean algebras is the same in the 
categories of Boolean algebras and of partial Boolean algebras. In particular, the terminal object $\mathbf{1}$ is the partial Boolean algebra with a single element $0=1$.

Coequalizers are harder to describe constructively, but the following theorem proves they do exist.

\section{Theorem 2 The category PBoolean is complete and cocomplete.}

Proof We are to show that PBoolean has coequalizers, i.e. that the diagonal functor $\Delta:$ PBoolean $\rightarrow$ PBoolean $^{(\bullet \rightrightarrows \bullet)}$ has a left adjoint (where $\bullet \rightrightarrows \bullet$ is the free category generated by the graph consisting of two vertices and two parallel arrows between them). Since we already know that PBoolean is complete and $\Delta$ preserves limits, Freyd's adjoint functor theorem shows that it suffices if the following solution set condition is satisfied [15, V.6]. For each $f, g: A \rightarrow B$ in PBoolean there is a setindexed family $h_{i}: B \rightarrow Q_{i}$ such that $h_{i} f=h_{i} g$, and if $h f=h g$ then $h$ factorizes through some $h_{i}$.

Take the collection of $h_{i}: B \rightarrow Q_{i}$ to comprise all 'quotients', i.e. (isomorphism classes of) surjections $h_{i}$ of partial Boolean algebras such that $h_{i} f=h_{i} g$. This collection is in fact a set. The proof is finished by observing that every morphism $h: B \rightarrow Q$ of partial Boolean algebras factors through (a surjection onto) its set-theoretical image, which is a partial Boolean subalgebra of $Q$, inheriting commeasurability from $B$ and algebraic operations from $Q$.

\subsection{Variations}

Results similar to those above hold for many classes of Boolean algebras, such as complete or countably complete Boolean algebras. For example, the former variation can be defined as follows.

Definition 2 A partial complete Boolean algebra consists of a partial Boolean algebra together with a (partial) operation

$$
\bigvee:\{X \subseteq A \mid X \times X \subseteq \odot\} \rightarrow A
$$

such that every set $S \subseteq A$ of pairwise commeasurable elements is contained in a set $T \subseteq A$, whose elements are also pairwise commeasurable, and on which the above operations determine a complete Boolean algebra structure. ${ }^{3}$

A morphism of partial complete Boolean algebras is a function that preserves commeasurability and all the algebraic structure, including $\bigvee$, whenever defined. We write PCBoolean for the resulting category.

A version of our main theorem also holds for such partial complete Boolean algebras, when we define a total subalgebra of a partial complete Boolean algebra to be a total subalgebra of the underlying partial Boolean algebra that is additionally closed under $\bigvee$.

\footnotetext{
${ }^{3}$ So $T$ is not only closed under $\neg, \wedge$ and $\vee$, but also under $\vee$.
} 
Theorem 3 Every partial complete Boolean algebra is a colimit of its total subalgebras.

Proof Completely analogous to Theorem 1.

\section{Stone Duality}

The full subcategory of PBoolean consisting of (total) Boolean algebras is just the category Boolean of Boolean algebras and their homomorphisms. This category is dual to the category of Stone spaces and continuous functions via Stone duality [10]:

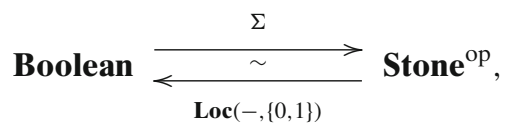

where $\Sigma(A)$ is the Stone spectrum of a Boolean algebra $A$. The dualizing object $\{0,1\}$ is both a locale and a (partial) Boolean algebra; recall that it is in fact the initial partial Boolean algebra $\mathbf{0}$.

One might expect that the category of partial Boolean algebras enters Stone duality (Eq. 1), and indeed the colimit theorem, Theorem 1, enables us to prove the following extension.

Proposition 2 There is a reflection

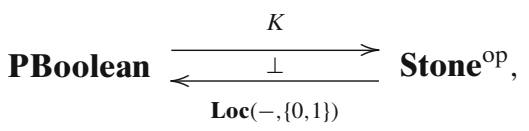

in which the functor $K$ is determined by $K(A)=\lim _{C \in \mathcal{C}(A)^{\mathrm{op}}} \Sigma(C)$.

Proof Let $A$ be a partial Boolean algebra and $X$ a Stone space. Then there are bijective correspondences:

$$
\frac{f: K(A)=\lim _{C \in \mathcal{C}(A)^{\text {op }} \Sigma(C) \rightarrow X}}{\frac{{ }_{C \in \mathcal{C}(A) \cdot f_{C}: \Sigma(C) \rightarrow X}}{\forall_{C \in \mathcal{C}(A) \cdot g_{C}: C \rightarrow \operatorname{Loc}(X,\{0,1\})}}} \begin{aligned}
& \text { (in Stone } \left.\text { Stone }^{\text {op }}\right) \\
& \text { (in Boolean) } \\
& \text { (in PBoolean) } A \rightarrow \operatorname{Loc}(X,\{0,1\})
\end{aligned}
$$

The first correspondence holds by definition of limit, the middle correspondence holds by Stone duality (Eq. 1), and the last correspondence holds by Theorem 1. Since all correspondences are natural in $A$ and $X$, this establishes the adjunction $K \dashv \operatorname{Loc}(-,\{0,1\})$. Finally, since a Boolean algebra is trivially a colimit of itself in PBoolean, the adjunction is a reflection. 
Theorem 4 The reflection $K \dashv \operatorname{Loc}(-,\{0,1\})$ extends Stone duality, i.e. the following diagram commutes (serially).

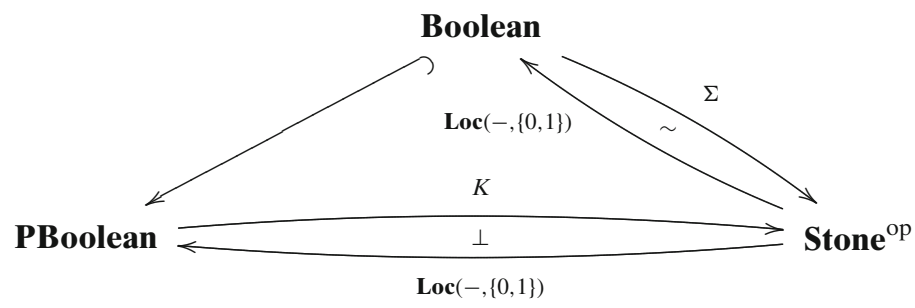

Proof If $A$ is a Boolean algebra, it is the initial element in the diagram $\mathcal{C}(A)^{\mathrm{op}}$ by Proposition 1(b). Hence $K(A)=\lim _{C \in \mathcal{C}(A)^{\text {op }}} \Sigma(C)=\Sigma(A)$.

Corollary 1 Boolean algebras form a reflective full subcategory of the category of partial Boolean algebras, i.e. the inclusion Boolean $\hookrightarrow$ PBoolean has a left adjoint $L:$ PBoolean $\rightarrow$ Boolean.

Proof The adjunctions of the previous theorem compose, giving the required left adjoint as $L=\operatorname{Loc}(-,\{0,1\}) \circ K$.

\section{Partial $C^{*}$-algebras}

The definitions of partial $C^{*}$-algebras and their morphisms closely resemble those of partial Boolean algebras. Indeed, both are instances of the partial algebras of Kochen and Specker, over the fields $\mathbb{Z}_{2}$ and $\mathbb{C}$, respectively. However, partial $\mathrm{C}^{*}$-algebras also have to account for a norm and involution, calling for some changes that we now spell out.

Definition 3 A partial $C^{*}$-algebra consists of a set $A$ with

- a reflexive and symmetric binary (commeasurability) relation $\odot \subseteq A \times A$;

- elements $0,1 \in A$;

- a (total) involution $*: A \rightarrow A$;

- a (total) function $\cdot: \mathbb{C} \times A \rightarrow A$;

- a (total) function $\|-\|: A \rightarrow \mathbb{R}$;

- $\quad$ (partial) binary operations $+, \cdot: \odot \rightarrow A$;

such that every set $S \subseteq A$ of pairwise commeasurable elements is contained in a set $T \subseteq A$, whose elements are also pairwise commeasurable, and on which the above operations determine the structure of a commutative $\mathrm{C}^{*}$-algebra. ${ }^{4}$

It follows from the last condition in the definition of a partial $\mathrm{C}^{*}$-algebra that commeasurable elements in a partial $\mathrm{C}^{*}$-algebra have to commute. In fact, as with

${ }^{4}$ This entails that $T$ contains 0 and 1 , is closed under all algebraic operations, and is norm-complete. 
partial Boolean algebras, partial $\mathrm{C}^{*}$-algebras whose commeasurability relation is total are nothing but commutative $\mathrm{C}^{*}$-algebras.

We again define the notion of a commeasurable or commutative subalgebra of a partial $\mathrm{C}^{*}$-algebra $A$ in the obvious way as a subset $T$ of $A$ of pairwise commeasurable elements on which the operations of $A$ determine a commutative $\mathrm{C}^{*}$-algebra structure. Also, if $S$ is subset of pairwise commeasurable elements, then again there must be a smallest commeasurable subalgebra $T$ that contains $S$ : simply take the intersection of all such subalgebras $T$. Alternatively, one can construct it as the set of those elements in $A$ that are limits of sequences whose terms are algebraic expressions involving the elements of $S$. We denote it by $A\langle S\rangle$.

The reader may be tempted to believe that every noncommutative $\mathrm{C}^{*}$-algebra can be regarded as a partial $C^{*}$-algebra by declaring that $a \odot b$ holds whenever $a$ and $b$ commute, but that would be incorrect. The reason for this is that we have required $\odot$ to be reflexive, so that $a a^{*}=a^{*} a$ holds for every element $a$ in a partial $\mathrm{C}^{*}$-algebra. Now, an element $a$ such that $a a^{*}=a^{*} a$ holds is called normal and it is not the case that every element in a $C^{*}$-algebra is normal.

What is true, however, is that one may regard the collection of normal elements of a $\mathrm{C}^{*}$-algebra as a partial $\mathrm{C}^{*}$-algebra by declaring two elements to be commeasurable whenever they commute. In fact, taking normal elements is part of a functor, if we consider the following class of morphisms of partial $\mathrm{C}^{*}$-algebras.

Definition 4 A partial *-morphism is a (total) function $f: A \rightarrow B$ between partial $\mathrm{C}^{*}$-algebras such that:

- $\quad f(a) \odot f(b)$ for commeasurable $a, b \in A$;

- $\quad f(a b)=f(a) f(b)$ for commeasurable $a, b \in A$;

- $\quad f(a+b)=f(a)+f(b)$ for commeasurable $a, b \in A$;

- $\quad f(a+i b)=f(a)+i f(b)$ for self-adjoint $a, b \in A$;

- $\quad f(z a)=z f(a)$ for $z \in \mathbb{C}$ and $a \in A$;

- $f(a)^{*}=f\left(a^{*}\right)$ for $a \in A$;

$-\quad f(1)=1$.

Partial $\mathrm{C}^{*}$-algebras and partial *-morphisms organize themselves into a category denoted by PCstar. ${ }^{5}$

Before we embark on proving that taking normal parts provides a functor from the category of $\mathrm{C}^{*}$-algebras to the category of partial $\mathrm{C}^{*}$-algebras, recall that an element $a$ of a $\mathrm{C}^{*}$-algebra is called self-adjoint when $a=a^{*}$, and that any element can be written uniquely as a linear combination $a=a_{1}+i a_{2}$ of two self-adjoint elements $a_{1}=\frac{1}{2}\left(a+a^{*}\right)$ and $a_{2}=\frac{1}{2 i}\left(a-a^{*}\right)$.

Proposition 3 There is a functor $N: \mathbf{C s t a r} \rightarrow \mathbf{P C s t a r}$ which sends every $C^{*}$-algebra to its normal part

$$
N(A)=\left\{a \in A \mid a a^{*}=a^{*} a\right\}
$$

\footnotetext{
${ }^{5}$ Most results hold for nonunital $C^{*}$-algebras, but for convenience we consider unital ones.
} 
which can be considered as a partial $C^{*}$-algebra by saying that $a \odot b$ holds whenever $a$ and $b$ commute. Moreover, $N$ is faithful and reflects isomorphisms and identities.

Proof The action of $N$ is well-defined on objects, since a subalgebra of a $\mathrm{C}^{*}$-algebra generated by a set $S$ is commutative iff the elements of $S$ are normal and commute pairwise.

On morphisms, $N$ acts by restriction and corestriction. To see that it has the properties the proposition claims it has, one uses the identity $a=a_{1}+i a_{2}$. For example, suppose $N(f)$ is surjective for a *-morphism $f: A \rightarrow B$ and let $b \in B$. Then there are $a_{1}, a_{2} \in N(A)$ with $f\left(a_{i}\right)=b_{i}$. Hence $f\left(a_{1}+i a_{2}\right)=b$, so that $f$ is surjective. Similarly, if $N(f)$ is injective, suppose that $f(a)=f\left(a^{\prime}\right)$. Then $f\left(a_{i}\right)=$ $f\left(a_{i}^{\prime}\right)$, and hence $a_{i}=a_{i}^{\prime}$, so that $a=a^{\prime}$ and $f$ is injective. Now, isomorphisms in (P)Cstar are bijective (partial) *-morphisms. Hence $f$ is an isomorphism when $N(f)$ is.

So therefore one way of thinking about a partial $\mathrm{C}^{*}$-algebra is as axiomatizing the normal part of a $\mathrm{C}^{*}$-algebra. Of course, we could have decided to drop the requirement that $\odot$ is reflexive, so that every $\mathrm{C}^{*}$-algebra would also be a partial $\mathrm{C}^{*}$-algebra, with commeasurability given by commutativity. We haven't done this for various reasons. First of all, we would like to have a notion given in terms of the physically relevant data. On Bohr's philosophy, which we adopt in this paper, the physically relevant information is contained in the normal part of a $\mathrm{C}^{*}$-algebra. Related to this is the fact that the Bohrification functor (which we will study in Section 7) only takes the normal part of a $\mathrm{C}^{*}$-algebra into account. Secondly, we wish to have a result saying how a partial $\mathrm{C}^{*}$-algebra is determined by its commutative subalgebras analogous to our result for partial Boolean algebras. With our present definition we do indeed have this result (it is Theorem 5 below), as we will now explain.

Denote by $\mathcal{C}:$ PCstar $\rightarrow$ POrder the functor assigning to a partial $\mathrm{C}^{*}$-algebra $A$ the collection of its commeasurable (i.e. commutative total) subalgebras $\mathcal{C}(A)$, partially ordered by inclusion. One immediately derives similar properties for the diagram $\mathcal{C}(A)$ in the category PCstar as for partial Boolean algebras.

Proposition 4 Let $A$ be a partial $C^{*}$-algebra.

(a) The least element of the poset $\mathcal{C}(A)$ is $A\langle 0\rangle=A\langle 1\rangle=\{z \cdot 1 \mid z \in \mathbb{C}\}$.

(b) The poset $\mathcal{C}(A)$ is filtered if and only if $A$ is a commutative $C^{*}$-algebra. In that case, $A$ is the largest element of the poset $\mathcal{C}(A)$.

We now prove the $\mathrm{C}^{*}$-algebra version of our main result.

Theorem 5 Every partial $C^{*}$-algebra is a colimit of its (finitely generated) commutative $C^{*}$-subalgebras.

Proof Let $A$ be a partial $\mathrm{C}^{*}$-algebra, and consider its diagram $\mathcal{C}(A)$ of (finitely generated) commutative $\mathrm{C}^{*}$-subalgebras $C$. Defining functions $i_{C}: C \rightarrow A$ by the 
inclusions yields a cocone in PCstar; we will prove that this cocone is universal. If $f_{C}: C \rightarrow B$ is another cocone, define a function $m: A \rightarrow B$ by

$$
m(a)=f_{A\left\langle a_{1}\right\rangle}\left(a_{1}\right)+i f_{A\left\langle a_{2}\right\rangle}\left(a_{2}\right) .
$$

This is a function satisfying $f_{C}=m \circ i_{C}$, and if it is a partial *-morphism then it is the unique such function, because $a_{1}$ and $a_{2}$ are uniquely defined by $a$. It remains to show that $m$ is a well-defined morphism of PCstar, which follows from the assumption that the $f_{C}$ are. Let us demonstrate the exemplaric clauses of preservation of scalar multiplication and multiplication. For $z \in \mathbb{C}$ and normal $a \in A$ we have $(z a)_{1}=x a_{1}-$ $y a_{2}$ and $(z a)_{2}=x a_{2}+y a_{1}$, where $z=x+i y$ for $x, y \in \mathbb{R}$. Hence

$$
\begin{aligned}
m(z a) & =f_{A\left\langle x a_{1}-y a_{2}\right\rangle}\left(x a_{1}-y a_{2}\right)+i f_{A\left\langle x a_{2}+y a_{1}\right\rangle}\left(x a_{2}+y a_{1}\right) \\
& =f_{A\left\langle a_{1}, a_{2}\right\rangle}\left(x a_{1}-y a_{2}\right)+i f_{A\left\langle a_{1}, a_{2}\right\rangle}\left(x a_{2}+y a_{1}\right) \\
& =x f_{A\left\langle a_{1}\right\rangle}\left(a_{1}\right)+i x f_{A\left\langle a_{2}\right\rangle}\left(a_{2}\right)+i y f_{A\left\langle a_{1}\right\rangle}\left(a_{1}\right)-y f_{A\left\langle a_{2}\right\rangle}\left(a_{2}\right) \\
& =(x+i y)\left(f_{A\left\langle a_{1}\right\rangle}\left(a_{1}\right)+i f_{A\left\langle a_{2}\right\rangle}\left(a_{2}\right)\right) \\
& =z m(a) .
\end{aligned}
$$

For commeasurable $a, b \in A$, we have $(a b)_{1}=a_{1} b_{1}-a_{2} b_{2}$ and $(a b)_{2}=a_{1} b_{2}+$ $a_{2} b_{1}$, so that

$$
\begin{aligned}
m(a b)= & f_{A\left\langle a_{1} b_{1}-a_{2} b_{2}\right\rangle}\left(a_{1} b_{1}-a_{2} b_{2}\right)+i f_{A\left\langle a_{1} b_{2}+a_{2} b_{1}\right\rangle}\left(a_{1} b_{2}+a_{2} b_{1}\right) \\
= & f_{A\left\langle a_{1} b_{1}-a_{2} b_{2}\right\rangle}\left(a_{1} b_{1}\right)-f_{A\left\langle a_{1} b_{1}-a_{2} b_{2}\right\rangle}\left(a_{2} b_{2}\right) \\
& +i f_{A\left\langle a_{1} b_{2}-a_{2} b_{1}\right\rangle}\left(a_{1} b_{2}\right)+i f_{A\left\langle a_{1} b_{2}-a_{2} b_{1}\right\rangle}\left(a_{2} b_{1}\right) \\
= & f_{A\left\langle a_{1}, b_{1}, a_{2}, b_{2}\right\rangle}\left(a_{1} b_{1}\right)-f_{A\left\langle a_{1}, b_{1}, a_{2}, b_{2}\right\rangle}\left(a_{2} b_{2}\right) \\
& +i f_{A\left\langle a_{1}, b_{1}, a_{2}, b_{2}\right\rangle}\left(a_{1} b_{2}\right)+i f_{A\left\langle a_{1}, b_{1}, a_{2}, b_{2}\right\rangle}\left(a_{2} b_{1}\right) \\
= & f_{A\left\langle a_{1}, b_{1}\right\rangle}\left(a_{1} b_{1}\right)+i f_{A\left\langle a_{1}, b_{2}\right\rangle}\left(a_{1} b_{2}\right)+i f_{A\left\langle a_{2}, b_{1}\right\rangle}\left(a_{2} b_{1}\right)-f_{A\left\langle a_{2}, b_{2}\right\rangle}\left(a_{2} b_{2}\right) \\
= & f_{A\left\langle a_{1}\right\rangle}\left(a_{1}\right) f_{A\left\langle b_{1}\right\rangle}\left(b_{1}\right)+i f_{A\left\langle a_{1}\right\rangle}\left(a_{1}\right) f_{A\left\langle b_{2}\right\rangle}\left(b_{2}\right) \\
& +i f_{A\left\langle a_{2}\right\rangle}\left(a_{2}\right) f_{A\left\langle b_{1}\right\rangle}\left(b_{1}\right)-f_{A\left\langle a_{2}\right\rangle}\left(a_{2}\right) f_{A\left\langle b_{2}\right\rangle}\left(b_{2}\right) \\
= & \left(f_{A\left\langle a_{1}\right\rangle}\left(a_{1}\right)+i f_{A\left\langle a_{2}\right\rangle}\left(a_{2}\right)\right)\left(f_{A\left\langle b_{1}\right\rangle}\left(b_{1}\right)+i f_{A\left\langle b_{2}\right\rangle}\left(b_{2}\right)\right) \\
= & m(a) m(b) .
\end{aligned}
$$

Together, Theorem 5 above and Theorem 6 below show that, up to partial *isomorphism, every $\mathrm{C}^{*}$-algebra can be reconstructed from its commutative $\mathrm{C}^{*}$ subalgebras, lending force to the Bohrification programme. In this light Theorem 5 could be said to embody a categorical crude version of complementarity.

Remark 2 We hasten to point out that Theorem 5 only works because of the way we have set things up. In particular, it would fail if we would drop the requirement that $\odot$ is reflexive. Also, it does not say that every $\mathrm{C}^{*}$-algebra is the colimit of its commutative subalgebras in the category Cstar, which would be false. 
The reason why Theorem 5 cannot be changed in these ways is that are nonisomorphic von Neumann algebras $A$ and $B$ for which $\mathcal{C}(A)$ and $\mathcal{C}(B)$ are isomorphic. (This follows from the work of Connes in [2]. For the experts, the argument is this: in [2] it is shown that there is a von Neumann algebra $A$ that is not anti-isomorphic to itself; since this $A$ is in standard form, i.e. has a separating cyclic vector, it follows that $A$ is not isomorphic to its commutant $A^{\prime}$. But Tomita-Takesaki theory shows that any such von Neumann algebra $A$ is anti-isomorphic to its commutant $A^{\prime}$, whence $\mathcal{C}(A) \cong \mathcal{C}\left(A^{\prime}\right)$.) Note that this, combined with Theorem 5, implies that there is a partial *-isomorphism $N(A) \rightarrow N(B)$ that is not of the form $N(f)$ for some $f: A \rightarrow B$. To put it another way, the faithful functor $N$ is not full.

Because of this the relevance of the current work to the theory of $C^{*}$-algebras proper is rather limited. But that is not our immediate aim: this paper primarily wishes to gain a better conceptual understanding of the Bohrification programme; and, as we have argued above, from that perspective the way we have set things up is very natural and Theorem 5 is a step forward.

We close this section with a discussion of completeness and cocompleteness properties of PCstar. It is known that the category of $\mathrm{C}^{*}$-algebras is both complete and cocomplete (for coproducts, see [16], and for coequalizers, see [8]). As it turns out, also PCstar is both complete and cocomplete.

PCstar is complete, as it has both equalizers and arbitrary products. Equalizers of partial $\mathrm{C}^{*}$-algebras are constructed as in Set, having inherited commeasurability and subalgebra structure. Products are given by $\prod_{i} A_{i}=\left\{\left(a_{i}\right)_{i} \mid a_{i} \in A_{i}, \sup _{i}\left\|a_{i}\right\|<\right.$ $\infty$, with componentwise commeasurability and algebraic structure. In particular, the terminal object $\mathbf{1}$ is the 0 -dimensional (partial) $\mathrm{C}^{*}$-algebra $\{0\}$, confusingly sometimes also denoted by 0 .

The coproduct of a family $A_{i}$ of partial $\mathrm{C}^{*}$-algebras is got by taking their disjoint union, identifying for every $z \in \mathbb{C}$ the elements of the form $z 1_{i}$. Notice that elements from different summands $A_{i}$ are never commeasurable in the coproduct. In particular, the initial object $\mathbf{0}$ is the 1 -dimensional (partial) $\mathrm{C}^{*}$-algebra $\mathbb{C}$, which is confusingly sometimes also denoted by 1 .

Coequalizers are harder to describe constructively, but they do exist.

\section{Theorem 6 The category PCstar is complete and cocomplete.}

Proof To show that PCstar has coequalizers, the same strategy as in the proof of Theorem 2 applies, because for every partial $\mathrm{C}^{*}$-algebra $A$ the collection of isomorphism classes of partial *-maps $f: A \rightarrow B$ such that $f(B)$ is dense in $A$ form a set and every partial $C^{*}$-algebra map with domain $A$ factors through a map of this form.

\subsection{Variations}

Theorem 5 holds for many varieties of (partial) $C^{*}$-algebras, as its proof only depends on (partial) algebraic properties. Let us consider (partial) Rickart $\mathrm{C}^{*}$-algebras as an example. Recall that a commutative $\mathrm{C}^{*}$-algebra $A$ is Rickart when every $a \in A$ has a unique projection $\mathrm{RP}(a) \in A$ such that $(1-\mathrm{RP}(a)) \cdot A$ is the right annihilator $\{b \in A \mid a b=0\}$. We call a partial C*-algebra $A$ together with a total map

$$
\mathrm{RP}: A \rightarrow A
$$


a partial Rickart $C^{*}$-algebra when every pairwise commeasurable $S \subseteq A$ is contained in a pairwise commeasurable $T \subseteq A$ on which the operations of $A$ yield a commutative Rickart $C^{*}$-algebra structure with RP's given by the function above. Denote the subcategory of PCstar whose objects are partial Rickart $\mathrm{C}^{*}$-algebras and whose morphism are partial *-morphisms that preserve RP by PRickart.

Theorem 7 Every partial Rickart $C^{*}$-algebra is the colimit of its commutative Rickart $C^{*}$-subalgebras.

Proof The proof of Theorem 5 holds verbatim when every reference to (partial) $\mathrm{C}^{*}$ algebras is replaced by (partial) Rickart C*-algebras.

If Rickart is the subcategory of Cstar consisting of Rickart $C^{*}$-algebras and *-morphisms preserving RP, then there is a functor

\section{$N:$ Rickart $\rightarrow$ PRickart}

sending every Rickart $C^{*}$-algebra $A$ to its normal part; this follows from [1, Proposition 4.4]. Similar results hold for any type of $C^{*}$-algebra that is defined by algebraic properties, such as $\mathrm{AW}^{*}$-algebras and spectral $\mathrm{C}^{*}$-algebras (see $\left.[9,5.1]\right)$. We will come back to $\mathrm{AW}^{*}$-algebras in Section 6 below.

The distinguishing feature of von Neumann algebras amongst $\mathrm{C}^{*}$-algebras, in contrast, is topological in nature. This makes it harder to come up with a notion of partial von Neumann algebra: the obvious definition-a partial $\mathrm{C}^{*}$-algebra $A$ in which every subset of commeasurable elements is contained in a von Neumann algebra-has the drawback that it is not clear if $N(A)$ would be a partial von Neumann algebra given a von Neumann algebra $A$. We can, however, still obtain the following.

Let $A$ be a von Neumann algebra. Without loss of generality, we may assume that $A$ acts on a Hilbert space $H$. Denote the von Neumann subalgebra of a von Neumann algebra $A$ generated by a subset $S \subseteq A$ by $A\langle\langle S\rangle$. It is the closure of the $\mathrm{C}^{*}$-algebra $A\langle S\rangle$ in the weak operator topology, and by von Neumann's double commutant theorem [12, Theorem 5.3.1], it equals $A\langle S\rangle^{\prime \prime}$.

Lemma 1 If a $C^{*}$-subalgebra $C$ of a von Neumann algebra $A$ is commutative, then so is its von Neumann envelope $A\langle\langle C\rangle$. Hence if $a \in A$ is normal, then $A\langle\langle a\rangle$ is commutative.

Proof Let $a, b \in C^{\prime \prime}$. Since $C^{\prime \prime}$ is the (weak operator) closure of $C$, we can write $b$ as a (weak operator) $\operatorname{limit} b=\lim _{n} b_{n}$ for $b_{n} \in C$. Then:

$$
\begin{array}{rlrl}
a b=a\left(\lim _{n} b_{n}\right) & =\lim _{n} a b_{n} & (\text { by }[12,5.7 .9(\mathrm{i})]) \\
& =\lim _{n} b_{n} a & & \left(\text { since } a \in C^{\prime \prime} \text { and } b_{n} \in C \subseteq C^{\prime}\right) \\
& =\left(\lim _{n} b_{n}\right) a & (\text { by }[12,5.7 .9(\mathrm{ii})]) \\
& =b a . &
\end{array}
$$


Theorem 8 Let $A$ be a von Neumann algebra acting on a Hilbert space. Then $N(A)$ is a colimit in PCstar of the (finitely generated) commutative von Neumann subalgebras of $A$.

Proof Using Lemma 1, the proof of Theorem 5 holds verbatim when every occurence of $A\langle S\rangle$ is replaced by $A\langle\langle S\rangle$.

\section{Gelfand Duality}

The full subcategory of PCstar consisting of commutative $\mathrm{C}^{*}$-algebras is just the category cCstar of commutative $\mathrm{C}^{*}$-algebras and *-morphisms. This category is dual to the category of compact Hausdorff spaces and continuous functions via Gelfand duality [10]. Constructively, the latter category is replaced by that of compact completely regular locales [3]:

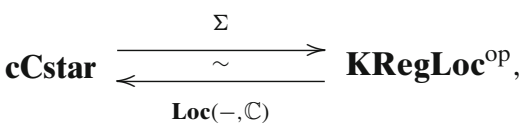

where $\Sigma(A)$ is the Gelfand spectrum of a commutative $\mathrm{C}^{*}$-algebra $A$. The dualizing object $\mathbb{C}$ is both a locale and a (partial) $\mathrm{C}^{*}$-algebra; recall that it is in fact the initial partial C*-algebra $\mathbf{0}$.

The colimit theorem, Theorem 5, together with the fact that the categories in Eq. 2 are cocomplete and complete, enables us to prove the following extension of Gelfand duality.

Proposition 5 There is a reflection

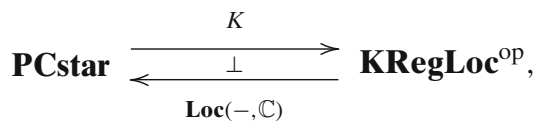

in which the functor $K$ is determined by $K(A)=\lim _{C \in \mathcal{C}(A)^{\mathrm{op}}} \Sigma(C)$.

Proof Let $A$ be a partial $\mathrm{C}^{*}$-algebra and $X$ a compact completely regular locale. Then there are bijective correspondences:

$$
\begin{aligned}
& f: K(A)=\lim _{C \in \mathcal{C}(A)^{\text {op }}} \Sigma(C) \rightarrow X\left(\text { in } \mathbf{K R e g L o c}^{\mathrm{op}}\right) \\
& \forall_{C \in \mathcal{C}(A)} \cdot f_{C}: \Sigma(C) \rightarrow X \quad\left(\text { in KRegLoc }{ }^{\text {op }}\right) \\
& \begin{array}{c}
\overline{\forall_{C \in \mathcal{C}(A) \cdot g_{C}: C \rightarrow \operatorname{Loc}(X, \mathbb{C})}} \quad \text { (in cCstar) } \\
g: A \rightarrow \operatorname{Loc}(X, \mathbb{C})
\end{array}
\end{aligned}
$$

The first correspondence holds by definition of limit, the middle correspondence holds by Gelfand duality (Eq. 2), and the last correspondence holds by Theorem 5 . Since all correspondences are natural in $A$ and $X$, this establishes the adjunction $K \dashv \operatorname{Loc}(-, \mathbb{C})$. Finally, since a commutative $C^{*}$-algebra is trivially a colimit of itself in PCstar, the adjunction is a reflection. 
Theorem 9 The reflection $K \dashv \operatorname{Loc}(-, \mathbb{C})$ extends Gelfand duality, i.e. the following diagram commutes (serially).

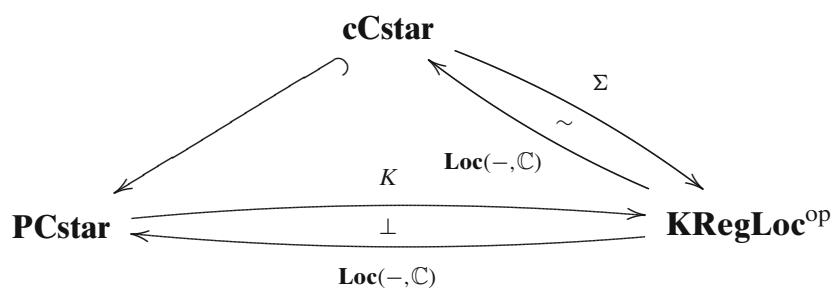

Proof If $A$ is a commutative $\mathrm{C}^{*}$-algebra, it is the initial element in the diagram $\mathcal{C}(A)^{\text {op }}$ by Proposition 4(b). Hence $K(A)=\lim _{C \in \mathcal{C}(A)^{\text {op }}} \Sigma(C)=\Sigma(A)$.

Corollary 2 Commutative $C^{*}$-algebras form a reflective full subcategory of partial $C^{*}$ algebras, i.e. the inclusion cCstar $\hookrightarrow$ PCstar has a left adjoint L: PCstar $\rightarrow$ cCstar.

Proof The adjunctions of the previous theorem compose, giving the required left adjoint as $L=\operatorname{Loc}(-, \mathbb{C}) \circ K$.

This means that for a partial $\mathrm{C}^{*}$-algebra $A$ one has

$$
\operatorname{PCstar}(A, \mathbb{C}) \cong \mathbf{c C s t a r}(L(A), \mathbb{C}) .
$$

In other words, multiplicative quasi-states of $A$ that are multiplicative on commutative subalgebras precisely correspond to states of $L(A)$. Thus these quasi-states have good (categorical) behaviour. However, things are not as interesting as they may seem. By the Kochen-Specker theorem, no von Neumann algebra $A$ without factors of type $I_{1}$ or $I_{2}$ can have such states ([4], see also [17]). It follows that $K(A)=\mathbf{0}$ and hence $L(A)=\mathbf{1}$ for such algebras. More generally, let us call a partial $\mathrm{C}^{*}$ algebra $A$ Kochen-Specker when $L(A)=\mathbf{1}$. Any such algebra $A$ has no quasi-states: $\operatorname{PCstar}(A, \mathbb{C}) \cong \mathbf{c C s t a r}(\mathbf{1}, \mathbb{C})=\emptyset$. Also, Kochen-Specker partial $\mathrm{C}^{*}$-algebras are a 'coproduct-ideal' in a sense that we now make precise. For $X \in \mathbf{K R e g L o c}$ we have $\mathbf{0} \times X=\mathbf{0}$, so by Gelfand duality (Eq. 2), we have $\mathbf{1}+C=\mathbf{1}$ for a commutative $\mathrm{C}^{*-}$ algebra $C$. So if $A \in \mathbf{P C s t a r}$ is Kochen-Specker, and $B \in \mathbf{P C s t a r}$ arbitrary, then also $A+B$ is Kochen-Specker:

$$
L(A+B)=L(A)+L(B)=\mathbf{1}+L(B)=\mathbf{1} .
$$

The first equality holds because $L$, being a left adjoint, preserves colimits. Nevertheless, the reflection of Theorem 9 is still interesting. Even though it does not teach much about the theory of $\mathrm{C}^{*}$-algebras proper, it is an important step in seeing how far $A$ can be reconstructed from $\mathcal{C}(A)$ (or its Bohrification $\underline{A}$, see Section 7 ). See also Remark 2.

\section{Projections, Partial AW*-algebras and Tensor Products}

This section discusses a functor PCstar $\rightarrow$ PBoolean, relating Sections 2 and 3 to Sections 4 and 5. 
6.1 Projections and Partial $\mathrm{AW}^{*}$-algebras

An element $p$ of a partial $C^{*}$-algebra $A$ is called a projection when it satisfies $p^{*}=$ $p=p^{2}$. The elements $0 \in A$ and $1 \in A$ are trivially projections; other projections are called nontrivial.

Lemma 2 There is a functor Proj: PCstar $\rightarrow$ PBoolean where $\operatorname{Proj}(A)$ is the set of projections of $A$.

Proof First, $\operatorname{Proj}(A)$ is indeed a partial Boolean algebra. Commeasurability is inherited from $A$. One easily checks that $\neg p=1-p$ is a projection when $p$ is. If $p, q$ are commeasurable in $\operatorname{Proj}(A)$, then they commute, whence the projection $p \wedge q=p q$ is also in $A[17,4.14]$. This makes $\operatorname{Proj}(A)$ into a partial Boolean algebra. Finally, morphisms of partial $\mathrm{C}^{*}$-algebras are easily seen to preserve projections, making the assignment $A \mapsto \operatorname{Proj}(A)$ functorial.

For the following class of partial $\mathrm{C}^{*}$-algebras we get stronger results.

Definition 5 A partial Rickart $\mathrm{C}^{*}$-algebra $A$ is a partial $A W^{*}$-algebra, if it comes equipped with an operation

$$
\bigvee:\{X \subseteq \operatorname{Proj}(A) \mid X \times X \subseteq \odot\} \rightarrow \operatorname{Proj}(A),
$$

in such a way that each pairwise commeasurable $S \subseteq A$ is contained in a pairwise commeasurable $T \subseteq A$ on which the operations determine a commutative $\mathrm{AW}^{*}$ algebra structure (i.e. the structure is that of a commutative Rickart $\mathrm{C}^{*}$-algebra, whose projections form a complete Boolean algebra with suprema given by the operation $\bigvee$ above). Denote the subcategory of PCstar whose objects are partial $\mathrm{AW}^{*}$-algebras and whose morphisms are partial *-morphisms which preserve RP and $\bigvee$ by PAWstar.

Lemma 3 The functor Proj restricts to a functor PAWstar $\rightarrow$ PCBoolean.

Proof Clear from the definition of a partial $\mathrm{AW}^{*}$-algebra.

Remark 3 It would be interesting to see whether this functor is part of an equivalence, like in the total case, where it is one side of an equivalence of categories between cAWstar and CBoolean.

Proposition 6 The functors Proj and $\mathcal{C}$ commute for partial $A W^{*}$-algebras: writing $\mathcal{C}^{\prime}$ for the functor PAWstar $\rightarrow$ [POrder, cAWstar], and $\mathcal{C}$ for the functor PBoolean $\rightarrow$ POrder, we have $\mathcal{C} \circ \operatorname{Proj}=\operatorname{Proj} \circ \mathcal{C}^{\prime}$. Explicitly,

$$
\{\operatorname{Proj}(C) \mid C \in \mathcal{C}(A)\}=\mathcal{C}(\operatorname{Proj}(A))
$$

for every partial $A W^{*}$-algebra $A$.

Proof This follows from the combination of Stone and Gelfand duality, which yields an equivalence between cAWstar and CBoolean. One direction of the equivalence 
is obtained by taking projections and the other is obtained by taking $C(X)$ where $X$ is the Stone space associated to the (complete) Boolean algebra. For the purposes of the proof, we will denote the latter composite functor by $F$. In particular, the projections $\operatorname{Proj}(C)$ of a commutative $\mathrm{AW}^{*}$-algebra $C$ form a complete Boolean algebra and the left-hand side is contained in the right-hand side.

For the converse, let $B$ be a complete Boolean lattice of projections in $A$. Then the projections in $B$ commute pairwise, and hence generate a commutative $\mathrm{AW}^{*}$ subalgebra $C=A\langle B\rangle$. We obviously have an inclusion $i: B \subseteq \operatorname{Proj}(C)$. But then the composite

$$
F B>\stackrel{F i}{\longrightarrow} F \operatorname{Proj}(C) \stackrel{\eta_{C}^{-1}}{\cong} C,
$$

where $\eta$ is the unit of the adjunction Proj $\dashv F$, shows that $F B$ is isomorphic to a commutative $\mathrm{AW}^{*}$-subalgebra of $A$ contained in $C$. This commutative subalgebra also contains $B$, because the diagram

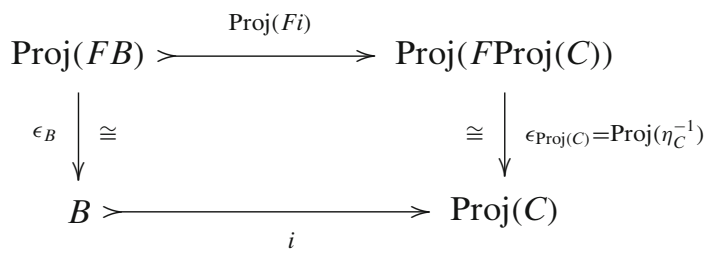

commutes by naturality of the counit $\epsilon: \operatorname{Proj}(F-) \Rightarrow 1$. Since $C=A\langle B\rangle$, this implies that $F i$ is an isomorphism. But then so is $i$ and therefore $B=\operatorname{Proj}(C)$.

As a corollary to the previous proposition, we can extend Proposition 4 with atoms to mirror Proposition 1. Keep in mind that the following corollary does not entail that $\mathcal{C}(A)$ is atomic.

Corollary 3 For a partial $A W^{*}$-algebra $A$, the atoms of the poset $\mathcal{C}(A)$ are $A\langle p\rangle$ for nontrivial projections $p$.

\subsection{Tensor Products}

It is clear from the description of coproducts in PCstar and PBoolean that the functor Proj preserves coproducts. Recall that in a coproduct of partial Boolean or $\mathrm{C}^{*}$ algebras, nontrivial elements from different summands are never commeasurable. Theorems 1 and 5 provide the option of defining a tensor product satisfying the adverse universal property.

Definition 6 Let $A$ and $B$ be a pair of partial Boolean algebras (partial C*-algebras). Define

$$
A \otimes B=\operatorname{colim}\{C+D \mid C \in \mathcal{C}(A), D \in \mathcal{C}(B)\},
$$

where $C+D$ is the coproduct in the category of Boolean algebras (commutative $C^{*}$-algebras). 
There are canonical morphisms $\kappa_{A}: A \rightarrow A \otimes B$ and $\kappa_{B}: B \rightarrow A \otimes B$ as follows. By definition, $A \otimes B$ is the colimit of $C+D$ for $C \in \mathcal{C}(A)$ and $D \in \mathcal{C}(B)$. Precomposing with the coproduct injections $C \rightarrow C+D$ gives a cocone $C \rightarrow A \otimes B$ on $\mathcal{C}(A)$. By the colimit theorem, $A$ is the colimit of $\mathcal{C}(A)$. Hence there is a mediating morphism $\kappa_{A}: A \rightarrow A \otimes B$.

The unit element for both the tensor product and the coproduct is the initial object 0. The big difference between $A \otimes B$ and the coproduct $A+B$ is that elements $\kappa_{A}(a)$ and $\kappa_{B}(b)$ are always commeasurable in the former, but never in the latter. Indeed, this universal property characterizes the tensor product.

Proposition 7 Let $f: A \rightarrow Z$ and $g: B \rightarrow Z$ be morphisms in the category PBoolean (PCstar). The cotuple $[f, g]: A+B \rightarrow Z$ factorizes through $A \otimes B$ if and only if $f(a) \odot f(b)$ for all $a \in A$ and $b \in B$.

Proof By construction, giving $h: A \otimes B \rightarrow Z$ amounts to giving a cocone $C+D \rightarrow$ $Z$ for $C \in \mathcal{C}(A)$ and $D \in \mathcal{C}(B)$. Because $C+D$ is totally defined, any morphism $C+$ $D \rightarrow Z$ must also be total. But this holds (for all $C$ and $D$ ) if and only if $f(a)$ and $g(b)$ are commeasurable for all $a \in A$ and $b \in B$, for (only) then can one take $h$ to be the cotuple of the corestrictions of $f$ and $g$.

The tensor products of Definition 6 makes Proj: PCstar $\rightarrow$ PBoolean a monoidal functor: the natural transformation $\operatorname{Proj}(A) \otimes \operatorname{Proj}(B) \rightarrow \operatorname{Proj}(A \otimes B)$ is induced by the cotuples $\operatorname{Proj}(C)+\operatorname{Proj}(D) \rightarrow \operatorname{Proj}(A \otimes B)$ of

$$
\operatorname{Proj}(C) \stackrel{\operatorname{Proj}(C \hookrightarrow A)}{\longrightarrow} \operatorname{Proj}(A) \stackrel{\operatorname{Proj}\left(\kappa_{A}\right)}{\longrightarrow} \operatorname{Proj}(A \otimes B)
$$

Proposition 8 The functor Proj: PCstar $\rightarrow$ PBoolean preserves coproducts and is monoidal.

We end this section by discussing the relation between the tensor products of partial Boolean algebras and those of Hilbert spaces, describing compound quantum systems. Let Hilb be the category of Hilbert spaces and continuous linear maps, and let $B:$ Hilb $\rightarrow$ PCstar denote the functor $B(H)=\mathbf{H i l b}(H, H)$ acting on morphisms as $B(f)=f \circ(-) \circ f^{\dagger}$ where $f^{\dagger}$ is the adjoint of $f$. The definition of the tensor product in PCstar as a colimit yields a natural transformation $B(H) \otimes B(K) \rightarrow$ $B(H \otimes K)$, induced by morphisms $C \rightarrow B(H \otimes K)$ for $C \in \mathcal{C}(B(H))$ given by $a \mapsto$ $a \otimes \mathrm{id}_{K}$. Initiality of the tensor unit $\mathbf{0}$ gives a morphism $\mathbf{0} \rightarrow B(\mathbb{C})$, and these data satisfy the coherence requirements. Hence the functor $B$ is monoidal, and therefore also the composite Proj $\circ B:$ Hilb $\rightarrow$ PBoolean is a monoidal functor.

\section{Functoriality of Bohrification}

The so-called Bohrification construction (see [9], whose notation we adopt) associates to every $\mathrm{C}^{*}$-algebra $A$ an internal commutative $\mathrm{C}^{*}$-algebra $\underline{A}$ in the topos $[\mathcal{C}(A)$, Set $]$, given by the tautological functor $\underline{A}(C)=C$. Gelfand duality then yields an internal locale, which can in turn be externalized. As it happens this construction 
works equally well for partial $\mathrm{C}^{*}$-algebras, so that Bohrification for ordinary $\mathrm{C}^{*}$ algebras can be seen as the composition of the functor $N$ from Proposition 3 with Bohrification for partial $\mathrm{C}^{*}$-algebras. Thus a locale is associated to every object of PCstar. In this final section we consider its functorial aspects. It turns out that the whole construction summarized above can be made into a functor from partial $\mathrm{C}^{*}$ algebras to locales by restricting the morphisms of the former.

Bohrification does not just assign a topos to each (partial) $\mathrm{C}^{*}$-algebra, it assigns a topos with an internal $\mathrm{C}^{*}$-algebra. To reflect this, we define categories of toposes equipped with internal structures.

Definition 7 The category RingedTopos has as objects pairs $(T, R)$ of a topos $T$ and an internal ring object $R \in T$. A morphism $(T, R) \rightarrow\left(T^{\prime}, R^{\prime}\right)$ consists of a geometric morphism $F: T^{\prime} \rightarrow T$ and an internal ring morphism $\varphi: R^{\prime} \rightarrow F^{*}(R)$ in $T$.

By CstaredTopos we denote the subcategory of RingedTopos of objects $(T, A)$ where $A$ is an internal $C^{*}$-algebra in $T$ and morphisms $(F, \varphi)$ where $\varphi$ is an internal *-ring morphism.

Notice that the direction of morphisms in this definition is opposite to the customary one in algebraic geometry [7, 4.1].

First of all, any functor $\mathbf{D} \rightarrow \mathbf{C}$ induces a geometric morphism $[\mathbf{D}$, Set $] \rightarrow[\mathbf{C}$, Set $]$, of which the inverse part is given by precomposition (see [11, A4.1.4]). We have already seen that $\mathcal{C}$ is a functor PCstar $^{\text {op }} \rightarrow$ POrder $^{\text {op }}$. Additionally, restricting a morphism $f: B \rightarrow A$ of partial $C^{*}$-algebras to $D \in \mathcal{C}(B)$ and corestricting to $\mathcal{C} f(D)$ gives a morphism of commutative $C^{*}$-algebras. Hence we obtain a geometric morphism of toposes $[\mathcal{C}(B)$, Set $] \rightarrow[\mathcal{C}(A)$, Set $]$ as well as an internal morphism of commutative *-rings. The latter is a natural transformation whose component at $D$ is $\underline{B}(D) \rightarrow\left((\mathcal{C} f)^{*} \underline{A}\right)(D)=\underline{A}(\mathcal{C} f(D))$. In other words, we have a functor PCstar ${ }^{\text {op }} \rightarrow$

\section{RingedTopos.}

In general, (inverse parts of) geometric morphisms do not preserve internal $\mathrm{C}^{*}$ algebras. But in this particular case, $(\mathcal{C} f)^{*} \underline{A}$ is in fact an internal $\mathrm{C}^{*}$-algebra in $[\mathcal{C}(B)$, Set $]$. The proof is contained in $[9,4.8]$, which essentially shows that any functor from a poset $P$ to the category of $\mathrm{C}^{*}$-algebras is always an internal $\mathrm{C}^{*}$-algebra in the topos $\left[P\right.$, Set]. Therefore, we really have a functor PCstar ${ }^{\text {op }} \rightarrow$ CstaredTopos, as the following proposition records.

\section{Proposition 9 Bohrification is functorial PCstar ${ }^{\text {op }} \rightarrow$ CstaredTopos.}

Applying internal Gelfand duality to $\varphi: \underline{B} \rightarrow(\mathcal{C} f)^{*} \underline{A}$ gives an internal locale morphism $\underline{\Sigma}\left((\mathcal{C} f)^{*} \underline{A}\right) \rightarrow \underline{\Sigma}(\underline{B})$. But to get a functor PCstar $^{\text {op }} \rightarrow$ LocaledTopos, $^{-}$ for the evident definition of the latter category, we would need an internal locale morphism $(\mathcal{C} f)^{*}(\underline{\Sigma}(\underline{A})) \rightarrow \underline{\Sigma}(\underline{B})$. As $(\mathcal{C} f)^{*}(\underline{\Sigma}(\underline{A}))$ and $\underline{\Sigma}\left((\mathcal{C} f)^{*} \underline{A}\right)$ are incomparable in general, this is where the current line of reasoning stops. The most natural way out is to restrict the morphisms of PCstar as follows.

Lemma 4 For morphisms $f: A \rightarrow B$ of PCstar, the following are equivalent:

(a) if $\mathcal{C} f(C) \leq D$ and $\mathcal{C} f\left(C^{\prime}\right) \leq D$ for $C, C^{\prime} \in \mathcal{C}(A)$ and $D \in \mathcal{C}(B)$, then there is $C^{\prime \prime} \in \mathcal{C}(A)$ such that $C \leq C^{\prime \prime}$ and $C^{\prime} \leq C^{\prime \prime}$ and $\mathcal{C} f\left(C^{\prime \prime}\right) \leq D$;

(b) $a \odot a^{\prime}$ when $f(a) \odot f\left(a^{\prime}\right)$. 
Proof First assume (a) and suppose $f(a) \odot f\left(a^{\prime}\right)$. Take $C=A\left\langle a, a^{*}\right\rangle, C^{\prime}=$ $A\left\langle a^{\prime},\left(a^{\prime}\right)^{*}\right\rangle$, and $D=B\left\langle f(a), f\left(a^{\prime}\right), f(a)^{*}, f\left(a^{\prime}\right)^{*}\right\rangle$. Then $\mathcal{C} f(C) \leq D$ and $\mathcal{C} f\left(C^{\prime}\right) \leq$ $D$. Hence there is $C^{\prime \prime}$ with $C \leq C^{\prime \prime}$ and $C^{\prime} \leq C^{\prime \prime}$. So $a, a^{\prime}$ are both elements of the commutative algebra $C^{\prime \prime}$, so $a \odot a^{\prime}$.

Conversely, assuming (b) and supposing $\mathcal{C} f(C) \leq D$ and $\mathcal{C} f\left(C^{\prime}\right) \leq D$, for all $a \in C$ and $a^{\prime} \in C^{\prime}$ we have $f(a), f\left(a^{\prime}\right) \in D$, so that $f(a) \odot f\left(a^{\prime}\right)$. But that means that $C$ and $C^{\prime}$ are commuting commutative subalgebras of $A$. Hence we can take $C^{\prime \prime}=A\left\langle C, C^{\prime}\right\rangle$.

We say that morphisms satisfying the conditions in the previous lemma reflect commeasurability. Notice that this class of morphisms excludes the type of counterexample discussed after Theorem 1 . To show how the assignment of a locale to a partial $\mathrm{C}^{*}$-algebra becomes functorial with these morphisms, let us switch to its external description $[9,5.16]$ :

$$
S(A)=\{F: \mathcal{C}(A) \rightarrow \text { Set } \mid F(C) \text { open in } \Sigma(C), F \text { monotone }\}
$$

For $A$ a partial $\mathrm{C}^{*}$-algebra, $S(A)$ is a locale. We want to extend this to a functor $S:$ PCstar $^{\text {op }} \rightarrow$ Loc, or equivalently, a functor $S:$ PCstar $\rightarrow$ Frm. Let $f: A \rightarrow B$ be a morphism of partial $C^{*}$-algebras, $F \in S(A)$, and $D \in \mathcal{C}(B)$. If $C \in \mathcal{C}(A)$ satisfies $\mathcal{C} f(C) \leq D$, then we have a morphism $C \rightarrow D$ given by the composition $C \stackrel{f}{\rightarrow} \mathcal{C} f(C) \leq D$. Its Gelfand transform is a frame morphism $\Sigma(C \stackrel{f}{\rightarrow} \mathcal{C} f(C) \leq$ $D): \Sigma(C) \rightarrow \Sigma(D)$. So, since $F(C) \in \Sigma(C)$, we get an open in $\Sigma(D)$. The fact that $\Sigma(D)$ is a locale allows us to take the join over all such $C$, ending up with the candidate action on morphisms

$$
S f(F)(D)=\bigvee_{\substack{C \in \mathcal{C}(A) \\ \mathcal{C} f(C) \leq D}} \Sigma(C \rightarrow \mathcal{C} f(C) \leq D)(F(C))
$$

Theorem 10 Bohrification gives a functor $S:$ PCstar $_{\mathrm{rc}}^{\mathrm{op}} \rightarrow$ Loc, where the domain is the opposite of the subcategory of PCstar of morphisms reflecting commeasurability.

Proof One quickly verifies that $S f(F)$, as given in Eq. 4 , is monotone, and hence a well-defined element of $S(B)$, and that $S f$ preserves suprema. The greatest element $1 \in S(A)$ is also preserved by $S f$ :

$$
S f(1)(D)=\bigvee_{\substack{C \in \mathcal{C}(A) \\ \mathcal{C} f(C) \leq D}} \Sigma(C \rightarrow D)(1)=\bigvee_{\substack{C \in \mathcal{C}(A) \\ \mathcal{C} f(C) \leq D}} 1=1
$$

The last equality holds because the join is not taken over the empty set: there is always $C \in \mathcal{C}(A)$ with $\mathcal{C} f(C) \leq D$, namely $C=\mathbf{0}$.

To finish well-definedness and show that $S f$ is a frame morphism, we need to show that it preserves binary meets. Recall that any frame satisfies the infinitary distributive law $\left(\bigvee_{i} y_{i}\right) \wedge x=\bigvee_{i}\left(y_{i} \wedge x\right)$. It follows that one always has $\left(\bigvee_{i} y_{i}\right) \wedge$ $\left(\bigvee_{j} x_{j}\right) \geq \bigvee_{k}\left(y_{k} \wedge x_{k}\right)$. A sufficient (but not necessary) condition for equality to hold would be if for all $i, j$ there exists $k$ such that $y_{i} \wedge x_{j} \leq y_{k} \wedge x_{k}$. Expanding 
the definition of $S f$ and writing $x_{C}=\Sigma(C \rightarrow D)(F(C))$ and $y_{C}=\Sigma(C \rightarrow D)(G(C))$ gives precisely this situation:

$$
\begin{aligned}
S f(F \wedge G)(D) & =\bigvee_{\mathcal{C} f\left(C^{\prime \prime}\right) \leq D} x_{C^{\prime \prime}} \wedge y_{C^{\prime \prime}} \\
(S f(F) \wedge S f(G))(D) & =\left(\bigvee_{\mathcal{C} f(C) \leq D} x_{C}\right) \wedge\left(\bigvee_{\mathcal{C} f\left(C^{\prime}\right) \leq D} y_{C^{\prime}}\right) .
\end{aligned}
$$

So, by Lemma 4, $S f$ will preserve binary meets if $f$ reflects commeasurability. Finally, it is easy to see that $S(\mathrm{id})=$ id and $S(g \circ f)=S g \circ S f$.

Let us conclude with four remarks concerning the last theorem.

- From the above proof it additionally follows that this choice of morphisms is the largest for which the theorem holds: PCstar $_{\mathrm{rc}}$ is the largest subcategory of PCstar for which Eq. 4 gives a well-defined frame morphism.

- Replacing the Gelfand spectrum by the Stone spectrum yields a similar functor PBoolean $_{\mathrm{rc}}^{\text {op }} \rightarrow$ Loc.

- The externalization of an internal locale in a topos $\operatorname{Sh}(L)$ consists of a locale morphism $L^{\prime} \rightarrow L$, and not just the locale $L^{\prime}$ itself. In that spirit, the previous theorem should have taken into account the map $S(A) \rightarrow \mathcal{C}(A)$ as well. To consider functorial dependence on $A$ of this map would require to change the category Loc into something more complicated, from which we refrain here.

- The category Loc is POrder-enriched, and hence a 2-category. We remark that the functor $S$ given by Eqs. 3 and 4 shows that the externalization of Bohrification is a two-dimensional colimit (in Loc) of the Gelfand spectra of commeasurable subalgebras. So, interestingly, whereas the one-dimensional colimit of the spectra will often be trivial because of the Kochen-Specker theorem (see the remarks at the end of Section 5), Bohrification shows that a two-dimensional colimit will be nontrivial.

Acknowledgements We thank Klaas Landsman for useful comments. Additionally, we owe the observation concerning [2] to Thierry Coquand, and the reference to [6] to John Harding and Mirko Navara and their forthcoming paper 'Subalgebras of orthomodular lattices'.

Open Access This article is distributed under the terms of the Creative Commons Attribution Noncommercial License which permits any noncommercial use, distribution, and reproduction in any medium, provided the original author(s) and source are credited.

\section{References}

1. Berberian, S.K.: Baer rings and Baer*-rings. Online book (2003). Available at http://www.ma. utexas.edu/mp_arc/c/03/03-181.pdf

2. Connes, A.: A factor not anti-isomorphic to itself. Ann. Math. 101(3), 536-554 (1975)

3. Coquand, T., Spitters, B.: Constructive Gelfand duality for C*-algebras. Math. Proc. Camb. Philos. Soc. 147(2), 323-337 (2009)

4. Döring, A.: Kochen-Specker theorem for Von Neumann algebras. Int. J. Theor. Phys. 44(2), 139-160 (2005)

5. Döring, A., Isham, C.J.: What is a thing?: topos theory in the foundations of physics. In: New Structures for Physics. Lecture Notes in Physics, vol. 813, pp. 753-937. Springer (2010) 
6. Grätzer, G., Koh, K.M., Makkai, M.: On the lattice of subalgebras of a Boolean algebra. Proc. Am. Math. Soc. 36, 87-92 (1972)

7. Grothendieck, A.: Le langage des schémas. In: Éléments de Géométrie Algébrique, vol. 4, pp. 5-228. Publications Mathématiques de l'IHÉS (1960)

8. Guichardet, A.: Sur la catégorie des algèbres de Von Neumann. Bull. Sciences Math., 2e Série 90, 41-64 (1966)

9. Heunen, C., Landsman, N.P., Spitters, B.: Bohrification. In: Deep Beauty-Understanding the Quantum World Through Mathematical Innovation. Cambridge University Press, Cambridge, UK (2010)

10. Johnstone, P.T.: Stone spaces. In: Cambridge Studies in Advanced Mathematics, vol. 3. Cambridge University Press, Cambridge, UK (1982)

11. Johnstone, P.T.: Sketches of an Elephant: a Topos Theory Compendium. Oxford University Press, London, UK (2002)

12. Kadison, R.V., Ringrose, J.R.: Fundamentals of the Theory of Operator Algebras. Academic, New York (1983)

13. Kalmbach, G.: Orthomodular Lattices. Academic, New York (1983)

14. Kochen, S., Specker, E.: The problem of hidden variables in quantum mechanics. J. Math. Mech. 17, 59-87 (1967)

15. Mac Lane, S.: Categories for the Working Mathematician, 2nd edn. Springer (1971)

16. Rainjonneau, D.: Existence des sommes dans certaines catégories d'algèbres. C. R. Acad. Sc. Paris, Série A 262, 283-285 (1966)

17. Rédei, M.: Quantum Logic in Algebraic Approach. Kluwer, Norwell (1998) 